\title{
Factores de riesgo psicosocial laboral en académicos de instituciones de educación superior en Latinoamérica: Una revisión sistemática
}

\section{Occupational Psychosocial Risk Factors in Academics of Higher Education Institutions in Latin America: a Systematic Review}

\author{
Alisma Monroy-Castillo \\ Universidad Autónoma del Estado de Morelos, Cuernavaca, México \\ ORCID: https://orcid.org/0000-0002-5965-694X \\ Arturo Juárez-García \\ Universidad Autónoma del Estado de Morelos, Cuernavaca, México \\ ORCID: https://orcid.org/0000-0003-3264-679X
}

Recibido 01-07-19 Revisado 25-07-19 Aprobado 10-09-19 En línea 18-09-19

*Correspondencia

Email: alisma.monroy@uaem.mx
Citar como:

Monroy-Castillo, A., \& Juárez-García, A. (2019). Factores de riesgo psicosocial laboral en académicos de instituciones de educación superior en Latinoamérica: Una revisión sistemática. Propósitos y Representaciones, 7(3), 248-272. doi: http://dx.doi.org/10.20511/pyr2019.v7n3.361 


\section{Resumen}

La presente revisión sistemática tuvo la finalidad de caracterizar los factores de riesgo psicosocial laboral y sus consecuencias en indicadores subjetivos y objetivos de salud en profesores universitarios de países hispanoparlantes de América Latina. Las búsquedas electrónicas se realizaron en PubMed, EBSCO, PsycINFO, PSICODOC, LILACS, MEDIGRAPHIC, IMBIOMED, Redalyc, Dialnet, Scielo y Doyma. Tres evaluadores seleccionaron de forma independiente los artículos para su revisión y evaluaron la calidad metodológica conforme la guía STROBE. 8 artículos cumplieron con los criterios de inclusión y exclusión. Los resultados confirmaron la presencia de diversos factores de riesgo psicosocial derivados de condiciones de trabajo vinculadas a las políticas internacionales de educación superior, y se muestran relaciones de estos factores con diversos efectos psicológicos y biológicos en la salud. Se discute sobre la necesidad de más estudios y de mejor calidad y la importancia de implementar programas de prevención de salud y bienestar en el sector académico.

Palabras clave: Estrés; Salud; Docentes; Universidades; América Latina.

\section{Summary}

The purpose of this systematic review was to characterize job psychosocial risk factors and their consequences on subjective indicators and health objectives in university professors from Spanish-speaking countries in Latin America. The web search included PubMed, EBSCO, PsycINFO, PSICODOC, LILACS, MEDIGRAPHIC, IMBIOMED, Redalyc, Dialnet, Scielo and Doyma. Three reviewers selected the articles for review independently and assessed their methodological quality according to the STROBE guideline. As a result, 8 articles met the inclusion and exclusion criteria. The results confirmed the presence of different psychosocial risk factors derived from working conditions linked to the higher education international policies. In the study, the relationships of these factors with different psychological and biological health effects are shown. The need for more and better quality studies and the importance of implementing health and welfare prevention programmes in the academic sector are also discussed.

Keywords: Stress; Health; Teachers; Universities, Latin America.

\section{Introducción}

En América Latina las Instituciones de Educación Superior (IES) han evolucionado de manera dinámica en las últimas décadas, y es a partir de los 80 's que las reformas a las políticas públicas educativas emitidas por el Banco Mundial (BM) y el Banco Interamericano de Desarrollo (BID) se han dirigido a las IES en particular, con el objeto de que incrementen su calidad educativa, sus capacidades en la expansión de la matrícula estudiantil de acuerdo a las necesidades de la región, y la diversificación de su oferta académica, aunque al mismo tiempo, con una tendencia inevitable a la contracción económica y la disminución de la planta académica (Millán, Calvanese y D’Aubeterre, 2017). Todo esto ha terminado creando esquemas burocráticos que obstaculizan el desarrollo de la educación superior, aunque reconociendo aparentemente sus leyes autonómicas y el apoyo del sector académico (Brunner, 2012).

Las estrategias implementadas y las metas de las políticas educativas para la IES, promueven la habilitación del profesorado, la generación e incremento de la productividad académica, y de programas acreditados; que pese a los lineamientos de reconocimiento y promoción del profesor, le han otorgado una condición de beneficio económico (ComasRodríguez y Rivera (2011). 
Las IES han incorporado los principios rectores para las Universidades de América Latina y el Caribe, declarados en la Conferencia Regional (CRES) y Mundial (CMES) sobre la Educación Superior de la UNESCO (2009; 2009), y la Organización de las Naciones Unidas para la Educación (1988), en la que se ponderaron: la "pertinencia social", así como la importancia de reforzar "la innovación, la interdisciplina y la transdisciplina" en las instituciones, y " el reconocimiento al personal docente y a los estudiantes como los protagonistas principales (...)" (Tünnermann, 2010, p.32). Todo lo anterior, para garantizar los niveles deseables en la enseñanza, la extensión, la investigación y la vinculación CRES-UNESCO, 2009), e impulsar procesos de evaluación interna y de acreditación por organismos externos. Sin embargo, estos quedaron enlazados a la lógica meritocrática, al financiamiento y presupuesto salarial, becas y estímulos, programas y proyectos institucionales; originando la deshomologación de los salarios (Izquierdo, 1998; López-Segrera, 2015), y convirtiendo las compensaciones económicas en ingresos que no impactan en la jubilación (Suárez \& Muñoz, 2016).

Todas estas transformaciones de las IES en Latinoamérica, caracterizadas por el énfasis en el control gubernamental a través de las acreditaciones, la privatización, la medición de la eficiencia productiva, la competitividad institucional y la excesiva vinculación con empresas privadas, han erosionado los principios sustantivos de la reforma universitaria de Córdova Argentina 1918, referidos a la autonomía universitaria, la co-gobernanza de todas las partes y la educación gratuita, entre otras (Torres \&Schugurensky, 2002; Díaz-Barriga, 2008; Taracena, 2011), lo que a su vez ha impactado en las condiciones de trabajo y derechos laborales de los trabajadores universitarios, aunque aparentemente cuenten con suficiente autonomía (Choi \& Juárez-García, 2019).

Se ha señalado frecuentemente que la política de la evaluación de la eficiencia productiva y del desempeño académico en particular, ha provocado la crisis actual de bienestar entre los profesores universitarios, como consecuencia de que dichos criterios son aplicados en los procedimientos tanto para su ingreso, como para su permanencia en las IES, y por el hecho de que dichas evaluaciones se ligan a su jerarquía ocupacional y su percepción salarial (Irigoyen \& Martínez, 2015; Martínez, Martínez \& Méndez, 2015; Martínez \& Preciado, 2009).

Asimismo, las IES de la región Latinoamericana también se han incorporado al sistema de "índices y rankings científico-académicos" organizados por organismos acreditadores nacionales e internacionales y cuyo eje medular es la "productividad científica" (Muñoz, 2019 p. 438); lo que genera que los académicos se conviertan en la parte medular y sostenimiento de las IES, aumentando no solo sus demandas y responsabilidades, sino privilegiando el individualismo y la competencia entre los académicos (Izquierdo, 1998).

Hasta ahora, es claro que todas estas políticas dirigidas a las IES, modificaron directamente el proceso del trabajo de los académicos, incidiendo en la diversificación de roles y el aumento de múltiples actividades académicas y cargas administrativas, la intensificación del trabajo y la prolongación de la jornada diaria que tienen que realizar para la consecución de indicadores de productividad y acreditación de programas educativos, lo que ha impactado a su vez en la disminución de horas de descanso y el tiempo de convivencia familiar (Sánchez y Martínez, 2014; Gómez, Perilla y Hermosa, 2015) y además, los expone a un esquema de criterios diferenciadores, que establece cada organismo evaluador (Martínez, Tobón \& Romero, 2017; Muñoz, 2019; Tünnermann, 2008).

De acuerdo con Martínez, Vega, Nava y Anguiano (2010), ser profesor universitario en América Latina, como actividad ocupacional, ejerce una mayor exposición al estrés por su función, organización social y demanda, y de acuerdo con Cladellas, (2008), entre las exigencias comunes se encuentran:

"El volumen de horas de trabajo, el ritmo al que se trabaja, los plazos, las exigencias y las presiones temporales impuestas en la ejecución de las tareas, los horarios que 
regulan la actividad laboral, los diversos aspectos temporales relacionados con la atención, la distracción y otros factores que influyen sobre el rendimiento, o la organización y la gestión del tiempo necesario para llevar a cabo las tareas (...)” (p. 239).

Todas estas condiciones laborales específicas del profesor universitario latinoamericano se traducen en la exposición a distintos factores de riesgo psicosocial, característicos del puesto y de la organización (Gil-Monte, 2009; Millán et al., 2017); y es indiscutible que dichas circunstancias generan detrimento en la salud física y emocional, así como en la calidad de la productividad y el desempeño académico de los profesores universitarios (Cisneros y Ramírez, 2009; Sánchez, 2017).

El efecto de los factores psicosociales laborales sobre las enfermedades depende en gran medida del estrés negativo (Hernández, Ortega \& Reidl, 2012; Juárez y Camacho, 2011), por lo que es importante definir cuáles son los factores de riesgo psicosocial que actualmente prevalecen como resultado del quehacer del profesor universitario (D’Aubeterre, Álvarez \& Ramírez, 2011) y cómo se vinculan a las funciones y a los criterios de evaluación, así como a las condiciones laborales y calidad de vida en general (Olmedo, Delgado, López, Yañez, Mora, Velasco \& Montero, 2013).

Existen estudios en Latinoamérica que identifican la presencia e impacto específico de los factores de riesgo psicosocial en el reporte subjetivo del distrés, la ansiedad generalizada, la depresión, y los trastornos del sueño de los académicos (García \& Muñoz, 2013; Sánchez \& Martínez, 2014), sin embargo pocos aún parecen haberse enfocado al impacto en indicadores más objetivos de desempeño o de enfermedades médicas que representan los grandes problemas de salud pública, tal es el caso del estudio de Heredia, Morales, Infante, Sánchez, Páez \& Gabini (2018) en Ecuador, el de Balcázar-Rueda, Gerónimo, Vicente-Ruíz, Hernández-Chávez, (2017) en México, o la investigación de Wilches-Luna, Hernández, Chavarro y Bernal-Sánchez, (2016) en Colombia, por citar algunos, los que demuestran su relación con enfermedades cardiovasculares, diabetes, índice de masa corporal y estilos de vida inadecuados.

No obstante, lo anterior, estos estudios escasos y aislados dejan ver la necesidad de hacer un balance sistemático y objetivo de la investigación disponible hasta hoy, que permita identificar la magnitud e impacto de los factores de riesgo psicosocial en la salud psicológica (indicadores subjetivos) y física (indicadores objetivos) de los docentes universitarios, en particular en la región latinoamericana, donde hay una usencia marcada en la literatura actual.

Con base en lo anterior, el objetivo del presente artículo fue realizar una revisión sistemática de las investigaciones para caracterizar los factores de riesgo psicosocial laboral y sus consecuencias en indicadores subjetivos y objetivos de salud en profesores universitarios de países hispanoparlantes de América Latina.

\section{Método}

De acuerdo con el proceso de realización de una revisión sistemática (Riley, et al., 2019; TorresFonseca y López-Hernández, 2014), las publicaciones se buscaron bajo los siguientes pasos predeterminados: 1) establecer inicialmente el objetivo de búsqueda, 2) definir criterios (palabras clave) de indagación reproducibles, 3) identificar estudios originales, 4) elegir artículos que describieran el diseño metodológico y 5) que presentaran los análisis e interpretación de los datos.

El procedimiento se llevó a cabo por tres revisores de manera independiente, en el período comprendido de enero 2014 a mayo de 2019 en: PubMed, EBSCO, PsycINFO, PSICODOC, LILACS, MEDIGRAPHIC, IMBIOMED, Redalyc, Dialnet, Scielo y Doyma; que son bases de datos de contenidos científicos más comunes en español que indexan revistas con publicaciones de la región latinoamericana. 
Se utilizaron los términos: "factores de riesgo psicosocial laboral", "factores psicosociales laborales", "evaluación", "salud", "profesores universitarios" y "docentes universitarios", los cuales se introdujeron de manera directa o en combinación para la identificación de publicaciones relevantes, así como para diferenciar los estudios que no eran objeto de la presente revisión (Figura 1).

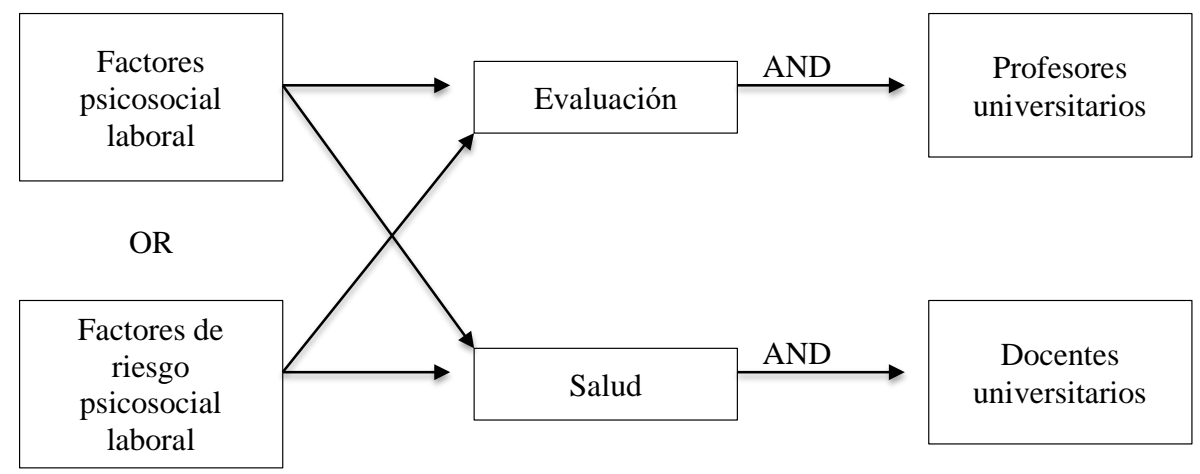

Figura 1. Palabras clave y operadores booleanos utilizados en la búsqueda de las publicaciones.

\section{Selección de los documentos}

Los artículos fueron seleccionados utilizando un primer filtro que correspondió al cumplimiento de los siguientes criterios de inclusión:

- Artículos originales empíricos publicados en revistas científicas y arbitradas cinco años atrás al inicio de la revisión (2014-2019).

- Publicaciones que mostraran que los estudios se realizaron con muestras de países hispanoparlantes de Latinoamérica.

- Textos completos que presentaran resultados de estudios dirigidos a profesores de nivel educativo superior, de instituciones públicas de algunos países Latinoamericanos.

- Artículos que incluyeran en su método de recolección de datos, evaluaciones de salud por auto-informe (subjetivas) y marcadores biológicos o indicadores objetivos para identificar el estado de salud de los profesores universitarios.

- Artículos publicados en español.

Como criterio de exclusión se establecieron:

- Artículos teóricos

- Docentes diferentes al nivel superior o de escuela privada

- Las publicaciones duplicadas

- Resúmenes de congresos, tesis o ponencias. 


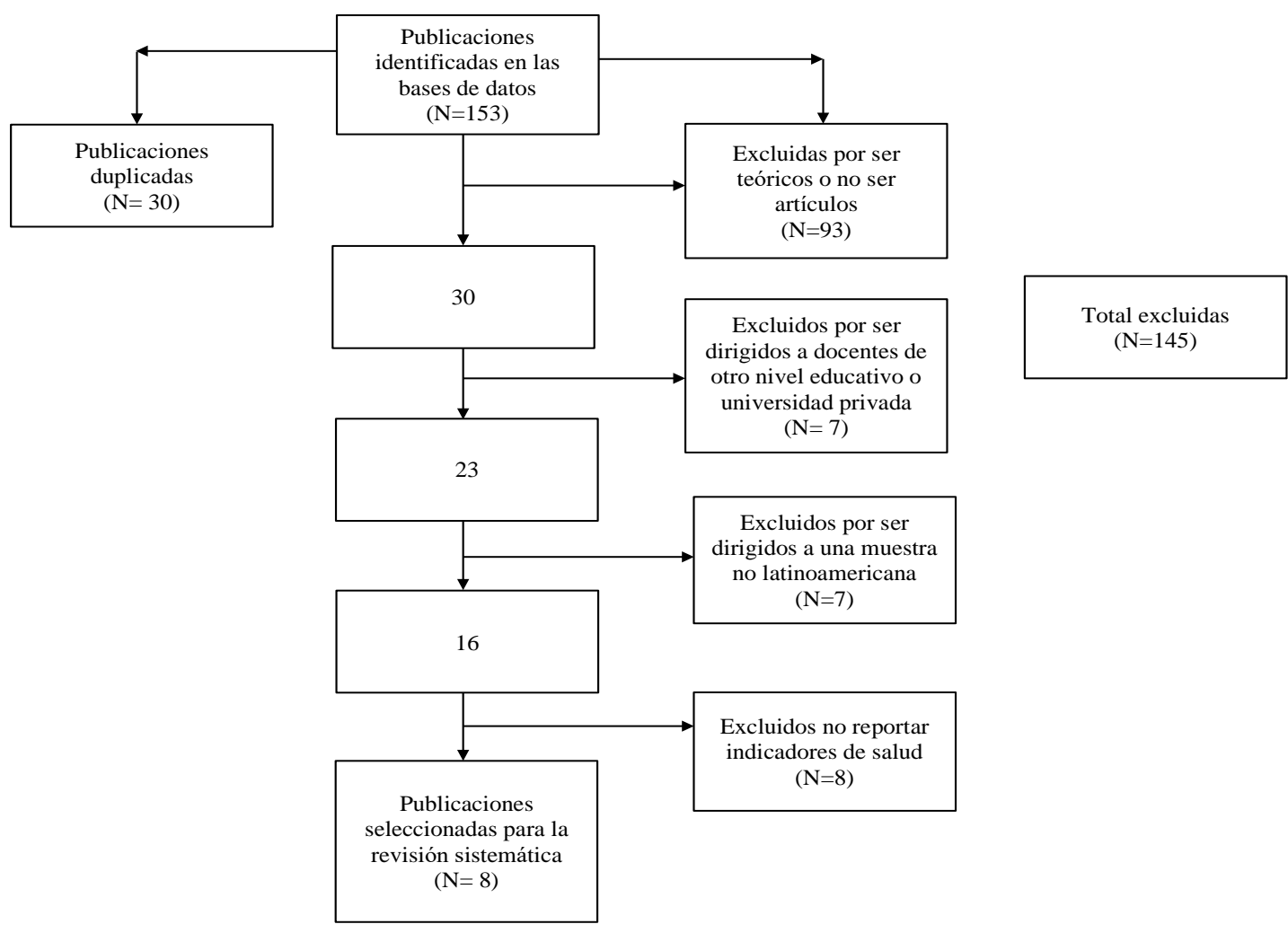

Figura 2. Proceso de selección de los artículos incluidos en la revisión.

\section{Calidad metodológica de las publicaciones}

En primer lugar, el contenido de las publicaciones se codificó de acuerdo a los descriptores de información propuestos por Jensen et al. (2011) que son: título, resumen, autor, año de publicación, diseño del estudio, características de la muestra, país, prevalencia y descriptivos de las variables de salud (p. ej. ansiedad, presión arterial, entre otros) y hallazgos relevantes.

Con los datos codificados en una base datos, y para garantizar la calidad de las publicaciones, se examinó el contenido de los artículos, utilizando la guía estándar de 22 puntos que comprende la Declaración Strengthening the Reporting of Observational studies in Epidemiology (STROBE) (Von Elm et al., 2008).

A cada uno de los temas de la guía, se le asignó una respuesta dicotómica (sí o no) para registrar sí el artículo revisado incluía o no ese criterio. El puntaje máximo para evaluar la calidad metodológica fue de 22 puntos.

En un tercer momento, un cuarto revisor verificó la información comprendida en la base de datos. Las discrepancias se resolvieron llevando a cabo diferentes reuniones con los evaluadores para cotejar la información, y asegurar el 100\% de concordancia.

\section{Resultados}

Los datos de cada publicación se resumen en las tablas 1 y 2 . Se seleccionaron 8 publicaciones que incluyeron en su muestra a profesores universitarios de IES públicas en países hispanoparlantes en Latinoamérica. El número de participantes en las investigaciones varió de 30 a 248 e incluyó a un total de 765 profesores universitarios. En relación con los datos sociodemográficos, solamente en tres investigaciones se reportó la edad de los participantes (estudios: 4, 6 y 8). El porcentaje de hombres (44.6\%) y mujeres (55.4\%) se describen en siete de 
los ocho estudios (estudios: 1, 2, 3, 4, 5, 6 y 8). La mayoría de las investigaciones que se eligieron se llevaron a cabo en México (87.5\%), seguidas por una de Colombia (12.5\%).

\section{Alcance de las investigaciones}

Las publicaciones seleccionadas para la presente revisión respondieron a los criterios de elegibilidad; sin embargo, hay una variabilidad en los aspectos metodológicos para evaluar la salud de los profesores universitarios como consecuencia de las condiciones laborales de las IES públicas (Tabla 1). El 100\% de los estudios son de enfoque cuantitativo, de diseño observacional y transversal. La descripción del contenido de los procedimientos e instrumentos para la recolección de datos (auto-informes o marcadores biológicos) variaron en función del objetivo y tamaño de la muestra.

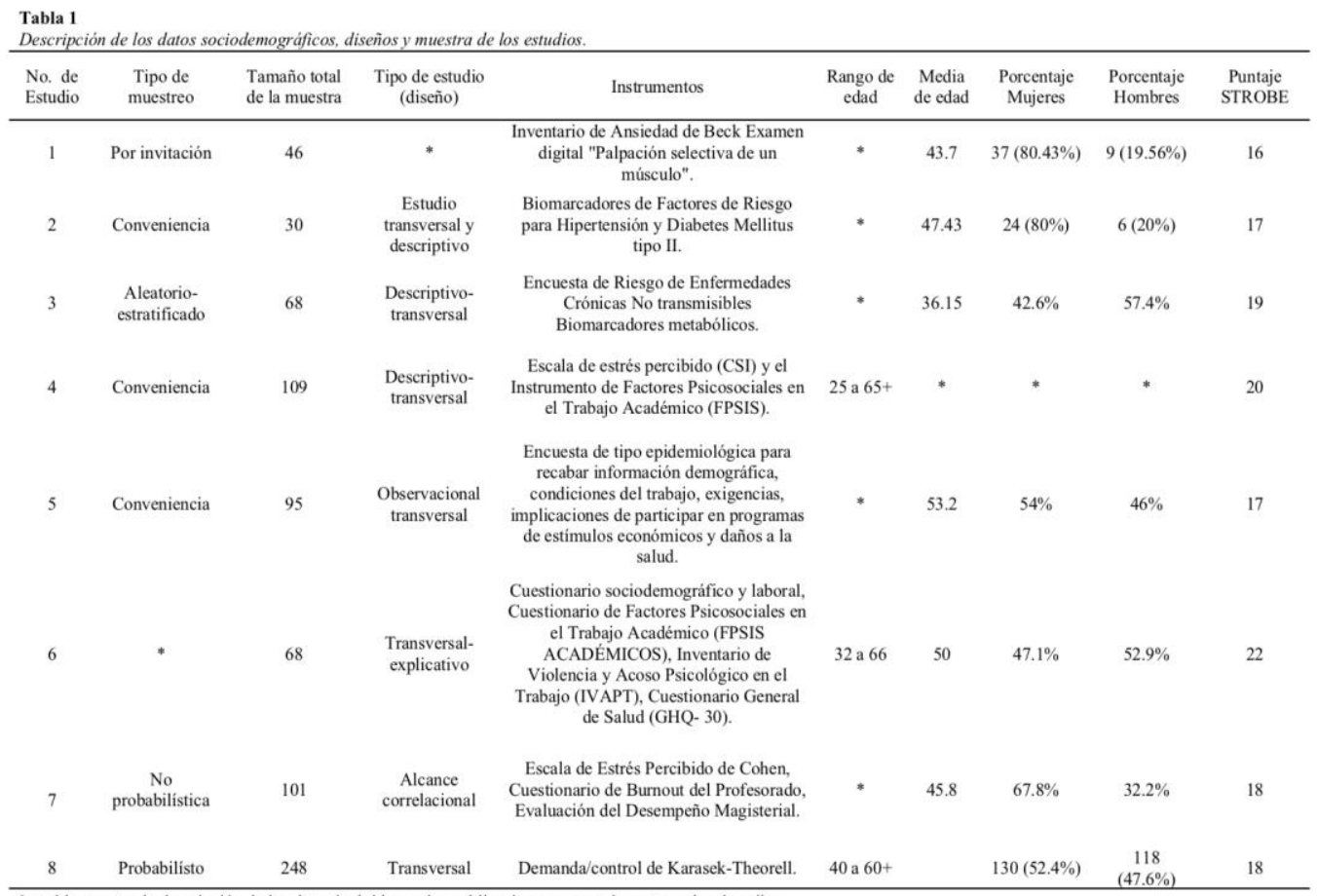

La tabla muestra la descripción de los datos incluidos en las publicaciones y como los autores los describen

"No reportado por los autores.

La mayoría de las evaluaciones indagaron sobre los factores de riesgo psicosocial del ámbito laboral de los académicos, mientras que tres de las investigaciones tuvieron un enfoque prioritariamente médico (estudios: 1, 2 y 3); en dos de las investigaciones se reportaron las valoraciones de marcadores biológicos para identificar síntomas fisiológicos en los profesores universitarios (p. ej. dolor miofascial y presión arterial) y en otra, se describió la prevalencia de Enfermedades Crónico No Trasmisibles (ECNT), determinadas por auto reporte e indicadores metabólicos. Las investigaciones que abarcaron evaluaciones psicológicas incluyeron mediciones de estrés (estudios: 4 y 7), ansiedad (estudio: 1), burnout (estudio: 7), entre otros (p ej. demanda/control de Karasek, estudio: 8). Paralelamente, se aplicaron instrumentos para identificar factores psicosociales de las condiciones laborales particulares de los profesores universitarios (estudios: 4, 5, 6 y 7) y, violencia y acoso psicológico (estudio: 6).

Asimismo, con la guía de verificación de la Declaración de la Iniciativa STROBE (Von Elm, et al., 2008) de 22 puntos para los estudios observacionales; se le asignó el puntaje a cada estudio por la descripción metodológica reportada en las publicaciones, que varió de 16 a 22 puntos (Tabla 1); tres de las ocho publicaciones no detallaron la metodología para la selección de la muestra (estudios: 6,7 y 8); otras, no describieron suficientemente los datos sociodemográficos de la muestra (p. ej. edad de la muestra, estudios: 1, 2, 3, 5 y 7). cuatro de las ocho publicaciones 
no informan el cálculo del tamaño de la muestra (estudios: 1, 5, 7 y 8); sin embargo, seis de los estudios reportaron que el tipo de muestreo que llevaron a cabo fue no probabilístico (estudios: 1, $2,4,5,7$ y 8$)$.

La puntuación del contexto/fundamentación, varía en relación de que los efectos se expliquen a partir de los factores psicosociales del trabajo (p. ej. estudio 5); o que sólo se evalúen indicadores de la salud de los profesores universitarios (como cualquier sector de trabajadores) dentro del marco de la medicina/salud pública (p. ej. estudio: 2).

Cuatro de los ocho estudios $(4,5,6$ y 7$)$ proporcionaron detalles precisos sobre los factores psicosociales laborales que se relacionan con problemas de salud como, la jornada laboral prolongada, en la cual reportaron que los docentes universitarios laboran más de 40 horas semanales, y que realizan trabajos en sus días de descanso o vacaciones, factores que se asociaron a trastornos músculo-esqueléticos.

Asimismo, la presencia de estrés por las condiciones laborales se relacionó al tipo de carrera, las exigencias del trabajo, aspectos de la organización, falta de apoyo social (entre compañeros y jefes), inconformidad con las recompensas (salario) y falta de reconocimiento y a las actividades derivadas de pertenecer a un programa de becas y/o estímulos. Además, cuando el estrés se ubicó en niveles altos por periodos prolongados se asoció a trastorno del sueño, ansiedad, disfonía, fatiga e indicadores cardio-metabólicos.

Finalmente, es importante destacar que aunque no hay suficiente información sobre los efectos negativos en la salud física y emocional de los académicos generados por las reformas a las políticas públicas educativas; las presentes publicaciones muestran evidencias sobre el impacto de las modificaciones a las condiciones laborales en las IES públicas de América Latina, por lo tanto, es necesario continuar estudiando los factores de riesgo psicosocial del trabajo de los profesores universitarios.

\section{Caracterización de factores de riesgo y salud.}

El presente estudio tuvo como propósito indagar el estado del arte que guardan las investigaciones sobre el efecto de los factores psicosociales laborales de los docentes de Universidades Públicas de América Latina (Tabla 2). De acuerdo con los resultados de la revisión, los factores que contribuyen al desarrollo de enfermedad son: la edad, sexo, el estilo de vida, las enfermedades heredofamiliares, además de la interacción con un medio ambiente laboral precario. En ese sentido, algunos autores señalan que las condiciones laborales, así como el ritmo de vida y a su vez, un estado emocional negativo crónico, juegan un rol importante, por ser factores de riesgo para la salud (Fernández et al., 2018; Martínez-Hernández, Enríquez, Moreno-Moreno y Martí, 2007).

Con respecto a las variables determinadas por los factores psicosociales laborales, las publicaciones destacaron que los docentes universitarios perciben la carga de trabajo, la participación en programas de estímulos (estudio: 4 y 8), el tipo de contrato con la institución (relación laboral) (estudios: 1 y 6), la jornada laboral prolongada (estudio: 4), la realización de actividades administrativas y técnicas (estudio: 4 y 5), la diferencia por área de conocimiento (p. ej. tipo de carrera) (estudio: 1) como variables de riesgo.

En relación con la prevalencia de variables de salud son, ansiedad (estudios: 1 y 5), dolor misofascial (estudio: 1), estrés (estudios: 4, 7 y 8) e hipertensión arterial (estudios: 2 y 3), entre otros. Entre las conclusiones de la mayoría de las publicaciones, señalaron las limitaciones por el tamaño de la muestra para ser concluyentes en los resultados. 
Tabla 2.

\begin{tabular}{|c|c|c|c|}
\hline $\begin{array}{l}\text { Base de datos } \\
\text { Artículo }\end{array}$ & Autores, País & Título & Principales prevalencias \\
\hline $\begin{array}{l}(1) \\
\text { Psyinfo }\end{array}$ & $\begin{array}{l}\text { García, C., Félix, R. O., Mercado, S. M., } \\
\text { Sabag, E., García, R., Mejía, D. y } \\
\text { Casanova, A. P. (2018). } \\
\text { México. }\end{array}$ & $\begin{array}{l}\text { Asociación entre el síndrome de dolor } \\
\text { miofascial y sintomatología ansiosa en } \\
\text { docentes de educación superior. }\end{array}$ & $\begin{array}{l}\text { Ansiedad y dolor miofascial mostraron una correlación significativa baja entre las dos variables }(\mathrm{r}=0.52, \mathrm{p}<0.000) \text {. } \\
\text { En relación a los subgrupos por tipo de contrato, la correlación fue significativa baja entre las dos variables }(\mathrm{r}=0.53 \text {, } \\
\mathrm{p}=0.04) \text { con profesores por contrato anual, }(\mathrm{r}=0.37 \text {, } \mathrm{p}=0.059) \text { con profesores de planta, }(\mathrm{r}=0.84, \mathrm{p}=0.069) \text { con } \\
\text { profesores auxiliares. En los subgrupos del tipo de carrera (Psicologia, Administración,, Educación y Contaduría y } \\
\text { Finanzas) se indicó una correlación significatitiva alta entre las dos variables }(\mathrm{r}=0.70, \mathrm{p}=0.03) \text { en docentes del área } \\
\text { de Contaduría, una correlación signnificativa moderada entre ambas variables }(\mathrm{r}=0.62, \mathrm{p}=0.008) \text { en docentes del área } \\
\text { de Psicologia, pero no en las otras carreras. }\end{array}$ \\
\hline $\begin{array}{c}(2) \\
\text { Mediagraphic }\end{array}$ & $\begin{array}{l}\text { Macías-Hernández, J. C., Alcantar- } \\
\text { Carrillo, O. E., Castro-Alcantar, M. G., } \\
\text { Kasten-Monges, M. J., y Cambero- } \\
\text { González, E. G. (2018). } \\
\text { México. }\end{array}$ & $\begin{array}{l}\text { Factores de riesgo para Hipertensión } \\
\text { Arterial Sistémica y Diabetes Mellitus Tipo } \\
2 \text { en el personal docente de uno de los } \\
\text { Departamentos del Centro Universitario de } \\
\text { Ciencias de la Salud. Universidad de } \\
\text { Guadalajarara, febrero-mayo } 2016 .\end{array}$ & $\begin{array}{l}\text { El estudio reportó que la mayoría de los } 30 \text { profesores tienen sobrepeso; entre los hallazgos se determinó que el } \\
73 \% \text { no realiza ejercicio, el } 36.7 \% \text { de tiene una dieta con bajo valor nutrimental y alto contenido calórico. El } 73.3 \% \\
\text { presenta indicadores de factores de riesgo de padecer DM2. El } 63.3 \% \text { manifestó sentirse estresado, variable que se } \\
\text { correlaciona con la TA sistólica y diastólica. Finalmente, se observó una alta correlación entre indicadores } \\
\text { metabólicos, sedentarismo, antecedentes heredofamiliares, y estilos de vida incrementa el riesgo para desarrollar } \\
\text { DM2 e HAS. }\end{array}$ \\
\hline $\begin{array}{c}(3) \\
\text { Mediagraphic }\end{array}$ & $\begin{array}{l}\text { López, J. A., Tenahua, I., Xicali, N., } \\
\text { Morales, F. A., Torres, A., y Posadas, G. } \\
\text { (2017). } \\
\text { México. }\end{array}$ & $\begin{array}{l}\text { Prevalencia de factores de riesgo } \\
\text { cardiovascular en académicos universitarios } \\
\text { de una institución pública de Oaxaca. }\end{array}$ & $\begin{array}{l}\text { La prevalencia de los factores de riesgo cardiovascular personales, se evidenció una prevalencia para sobrepeso de } \\
42.6 \% \text {, obesidad } 17.6 \% \text {, hipercolesterolemia } 35.3 \% \text {, tabaquuismo } 11.7 \% \text { y diabetes mellitus } 8.8 \% \text {. En relación al } \\
\text { estilo de vida de los académicos; se registró que } 64.7 \% \text { de participantes que no realizan actividades fisicas y } \\
\text { permanecen sentados en un día hábil hasta un promedio de } 5.09 \text { hrs. al día. }\end{array}$ \\
\hline $\begin{array}{c}(4) \\
\text { Mediagraphic }\end{array}$ & $\begin{array}{l}\text { Rodríguez-Vega, M. C., Preciado, M. L., } \\
\text { Aguilar-Aldrete, M. E., Aranda-Beltrán, } \\
\text { C., Leén-Cortés, S., y Franco, S. A. } \\
\text { (2018). } \\
\text { México. }\end{array}$ & $\begin{array}{l}\text { Causas y situaciones que inciden en el estrés } \\
\text { laboral de profesores del CUCS-U DE G. }\end{array}$ & $\begin{array}{l}\text { En relación a las condiciones laborales se identificicó que docentes tienen una jornada laboral de } 11 \text { a } 20 \text { horas a la } \\
\text { docencia, mientras que los profesores investigadores se dedican de una a } 10 \text { hrs. a la docencia y actividades } \\
\text { administrativas, de } 21 \text { a } 30 \text { horas por semana a la investigación. La prevalencia de estrés en profesores es de } 82.6 \% \text {. } \\
\text { Los factores psicosociales que favorecen el estrés son las condiciones del lugar de trabajo ( }(95.4 \%) \text {, la interacción } \\
\text { social y los aspectos organizacionales }(72.5 \%) \text { y la carga de trabajo }(66.1 \%) \text {. }\end{array}$ \\
\hline $\begin{array}{l}(5) \\
\text { Redalyc }\end{array}$ & $\begin{array}{l}\text { Sánchez, C., y Martínzz, S. (2014). } \\
\text { México. }\end{array}$ & $\begin{array}{l}\text { Condiciones de trabajo de docentes } \\
\text { universitarios, satisfacción, exigencias } \\
\text { laborales y daños a la salud. }\end{array}$ & 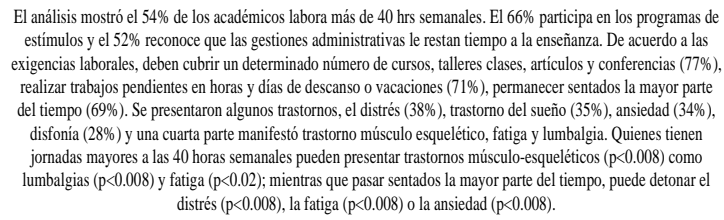 \\
\hline $\begin{array}{c}(6) \\
\text { Redalyc }\end{array}$ & $\begin{array}{l}\text { Acosta-Fernández, M., Parra-Osorio, L., } \\
\text { Restrepo-García, J., Pozos-Radillo, B., } \\
\text { Aguilera-Velasco, M., Torres-López, T. } \\
\text { (2017). } \\
\text { Colombia. }\end{array}$ & $\begin{array}{c}\text { Condiciones psicosociales, violencia y salud } \\
\text { mental en docentes de medicina y } \\
\text { enfermería. }\end{array}$ & $\begin{array}{l}\text { La presencia de condiciones psicosociales negativas (nivel medio, } 66 \%) \text { se asoció para ambos las carreras de } \\
\text { enfermería y medicina, con una probabilidad alta de presencia de situaciones y comportamientos de violencia } \\
\text { p }<0.000 \text {. Respecto a los FPSIS Académicos, se destacaron las exigencias laborales }(44.1 \%) \text { y remuneración al } \\
\text { rendimiento }(20.6 \%) \text {, además de inconformidade insatisfacción con el salario. Entre las condiciones de mayor } \\
\text { efecto negativo son los factores psicosociales como el acoso psicológico. }\end{array}$ \\
\hline $\begin{array}{l}\text { (7) } \\
\text { Redalyc }\end{array}$ & $\begin{array}{l}\text { Cárdenas, M., Méndez, L., y González } \\
\text { Ramírez, M. (2014). } \\
\text { México. }\end{array}$ & $\begin{array}{l}\text { Evaluación del desempeño docente, estrés y } \\
\text { burnout en profesores universitarios. }\end{array}$ & $\begin{array}{l}\text { Se encontró una correlación negativa significativa entre falta de realización y cambio en el desempeno docente ( } \mathrm{r}=- \\
\text {.446; } \mathrm{p}=0.003) \text {. La correlación entre los dos aspectos de desorganización institucional, supervisión y condiciones } \\
\text { organizacionales, con cambio en desempeño docente, indicaron que, a medida que se percibe más desorganización } \\
\text { institucional, el desempeño docente empeora ( } \mathrm{r}=361, \mathrm{p}=.019 \text { y r=.308, } \mathrm{p}=0407 \text {; respectivamente). }\end{array}$ \\
\hline $\begin{array}{l}(8) \\
\text { Scielo }\end{array}$ & $\begin{array}{l}\text { Palacios, M. E., y Montes de Oca, V. } \\
\text { (2017). } \\
\text { México. }\end{array}$ & $\begin{array}{l}\text { Condiciones de Trabajo y Estrés en } \\
\text { Académicos Universitarios. }\end{array}$ & $\begin{array}{l}\text { Del total de la muestra, } 76 \% \text { participa en el Programa de Primas al Desempeño del Rendimiento Académico } \\
\text { (PRIDE) y } 34 \% \text { pertenece al Sistema Nacional de Investigadores (SNI). El puntaje mostró que el } 62 \% \text { consideraba } \\
\text { tener alto control en su trabajo. De manera similar, } 65 \% \text { reportó altas exigencias o demandas y } 55 \% \text { contar con el } \\
\text { apoyo de jefes y compañeros. El 19.4\% del total de académicos tuvo alta tensión o estrés en el trabajo, producto de } \\
\text { la combinación bajo control y alta demanda. En relación a los marcadores biológicos, el } 40 \% \text { reportó que tenían } \\
\text { colesterol alto y 32\% triglicéridos por arriba de los límites normales. }\end{array}$ \\
\hline
\end{tabular}

\section{Discusión}

El objetivo del presente artículo fue realizar una revisión sistemática de las investigaciones para caracterizar los factores de riesgo psicosocial laboral y sus consecuencias en indicadores subjetivos y objetivos de salud en profesores universitarios de países hispanoparlantes de América Latina, y un primer hallazgo interesante de discutir es la escasa presencia de estudios, pues la revisión alcanzó solo ocho investigaciones. Esto confirma la necesidad de mayor investigación empírica al respecto, pues la mayor parte de los artículos excluidos fue por su enfoque meramente teórico, lo que también resultan importantes, pero necesario equilibrar con evidencia empírica.

Asimismo, parece limitada también la visión inter o transdisciplinaria, pues siguen siendo pocos los estudios que incluyen indicadores de salud objetivos o biomarcadores, los que sirven para comprender de manera más integral los complejos procesos del estrés y las afectaciones en el proceso salud enfermedad de los docentes universitarios. Este es un aporte en la presente revisión, que supera revisiones previas que abordan solo el enfoque psicológico-subjetivo del estrés o burnout (Diehl \& Marin, 2016; Gardner, 2010; Watts \& Robertson, 2011). 
Los resultados también permiten identificar la necesidad futura de mejorar la calidad de investigación, pues aún los estudios seleccionados que pasaron por filtros tuvieron diseños no experimentales (observacionales)- transversales, y con muestras seleccionadas por conveniencia que no pasaban de los 110 participantes (en 7 de 8 estudios), lo que aleja de conclusiones o interpretaciones causales sobre las relaciones encontradas y su generalización a la población.

No obstante, lo anterior, la presente revisión de los estudios seleccionados confirma la presencia de factores psicosociales laborales de los docentes universitarios y su relación con daños a su salud tanto a nivel subjetivo como objetivo. En particular, la revisión muestra la presencia de estresores psicosociales tales como el tipo de carrera, el tipo de contrato, carga de trabajo, jornadas largas, aspectos de la organización, falta de apoyo social (entre compañeros y jefes), inconformidad con las recompensas (salario), falta de reconocimiento y demandas derivadas de pertenecer a un programa de becas y/o estímulos. Estos se encuentran claramente vinculados a las condiciones de trabajo creadas por las recientes políticas institucionales antes mencionadas, donde se prioriza la productividad científica individual basada en indicadores de competitividad, acreditación y otras iniciativas burocrácticas. Además, cuando el estrés se ubica en niveles altos por periodos prolongados en los docentes universitarios, esto se asocia a trastornos del sueño, ansiedad, disfonía, fatiga, dolor misofacial, hipertensión arterial e indicadores cardiometabólicos, en los estudios seleccionados.

La suma de este tipo de evidencias, coadyuvan a promover la necesidad de transformar favorablemente los procesos existentes para aminorar los efectos negativos del ámbito laboral de los docentes universitarios; impulsando estrategias para desarrollar políticas públicas y programas para generar las condiciones que mejoren la estructura Universidad Pública-Profesor Universitario, una relación que particularmente en Latinoamérica ha mostrado el rezago en el avance del cumplimiento de las normas y recomendaciones que regulan la Seguridad y Salud en el Trabajo emitidas por organismos internacionales. De aquí la necesidad de implementar programas de prevención y promoción de bienestar en el docente universitario, independientemente del avance en la investigación.

Es necesario generar políticas de condiciones que no interfieran con la autonomía de las IES, pero al mismo tiempo que no reduzcan, o eliminen la responsabilidad compartida de dichos efectos, con los organismos acreditadores y evaluadores de indicadores, que basan sus políticas en esquemas administrativo-financieros; además de "la participación de distintos actores sociales en la definición de prioridades y políticas educativas, así como en la evaluación de éstas" (Conferencia Regional de la Educación Superior en América Latina y el Caribe, [CRES] 2009 p.96).

La revisión confirma que existe poca información sobre investigaciones dirigidas a profesores universitarios en América Latina que den cuenta de los factores de riesgo psicosocial laboral y sus efectos en el sector académico universitario; lo que según Cisneros y Ramírez (2009) es prioritario: investigar más sobre las interacciones de las condiciones laborales y exigencias psicológicas particulares a las que responden los profesores universitarios, ya que esto permitirá identificar el mecanismo de las enfermedades ocupacionales, sus costos e implicaciones en el cumplimiento de su misión educativa. Es necesario también identificar factores de riesgo psicosocial más específicos de este sector, e incorporar algunas variables que corresponden a las funciones particulares de los académicos de Universidades Públicas Latinoamericanas, con la finalidad de crear un precedente sobre los mecanismos que provocan el deterioro de la salud de este sector educativo.

El interés de las IES públicas se ha mantenido en la continua creación, y la verificación del cumplimiento de indicadores (Carot, 2012), que no consideran las condiciones específicas de cada país y de la realidad actual a la que se enfrentan los académicos. Las ocho publicaciones seleccionadas contribuyen al tema de los efectos a la exposición permanente de los factores de riesgo psicosocial laboral de académicos del nivel superior educativo; no obstante, el alcance y 
limitaciones metodológicas de las investigaciones, todavía no responden una cuestión fundamental: ¿cuál es el costo de la excelencia académica institucional y el cumplimiento de indicadores de productividad poniendo en riesgo la integridad psicosocial de los profesores universitarios?. Por tal motivo, se sugieren más investigaciones futuras incluyan diseños prospectivos, así como la evaluación de marcadores biológicos y psicológicos, que permitan explicar qué factores de riesgo inciden en la salud física y emocional.

\section{Referencias}

Acosta-Fernández, M., Parra-Osorio, L., Restrepo-García, J., Pozos-Radillo, B., AguileraVelasco, M., \& Torres-López, T. (2017). Condiciones psicosociales, violencia y salud mental en docentes de medicina y enfermería. Salud Uninorte, 33(3), 344-354.

Balcázar-Rueda, E., Gerónimo, E., Vicente-Ruíz, M. A., \& Hernández-Chávez, L. (2017). Factores de riesgo cardiovascular en docentes universitarios de ciencias de la salud. Salud Quintana Roo, 10(37), 7-12.

Brunner, J. J. (2012). La idea de universidad en tiempos de masificación. Revista iberoamericana de educación superior, 3(7), 130-145.

Cárdenas, M., Méndez, L., \& González Ramírez, M. (2014). Evaluación del desempeño docente, estrés y burnout en profesores universitarios. Revista Electrónica Actualidades Investigativas en Educación, 14(1), 1-22.

Carot, J. M. (2012). Sistema básico de indicadores para la educación superior de América Latina. Editorial Universitat Politécnica de Valéncia.

Choi, B., y Juárez-García, A. (2019). Positive association between job decision authority and systolic blood pressure: a statistical artifact? Scandinavian Journal of Work, Environment \& Health, 45(2), 209-210.

Cisneros y Ramírez, M. (2009). Prevalencia de enfermedades en trabajadores académicos de una universidad pública según seguro de gastos médicos. Revista Salud de los trabajadores. Volumen 17(2), 121-131.

Cladellas, R. (2008). La ausencia de gestión de tiempo como factor de riesgo psicosocial en el trabajo. Intangible Capital, 4 (4), 237-254.

Comas-Rodríguez, O. \& Rivera, A. (2011). La docencia universitaria frente a los estímulos económicos. Educación, Sociedad y Cultura, 32, 41-54.

Conferencia Regional de la Educación Superior en América Latina y el Caribe. (2009). Conferencia Regional de la Educación Superior en América Latina y el Caribe (CRES). Declaraciones y plan de acción. Perfiles educativos, 31(125), 90-108.

D’Aubeterre, M. E., Álvarez, J. C., \& Ramírez, T. (2011). Construcción de un inventario de percepción de estresores en docentes universitarios (IPED-U). En C. Blanco (Comp.). Investigación Educativa: Venezuela en Latinoamérica Siglo XXI-Parte II, 93-115. Caracas: Centro de Investigaciones Educativas de la Escuela de Educación de la Universidad Central de Venezuela.

Díaz-Barriga, A. F. (2008). Temas de debate en la innovación educativa. E. Lugo (comp.), Reformas Universitarias: su impacto en la innovación curricular y la práctica docente, México, UAEM/ANUIES, 23-38.

Diehl, L., \& Marin, A. H. (2016). Adoecimento mental em professores brasileiros: revisão sistemática da literatura. Estudos Interdisciplinares em Psicologia, 7(2), 64-85.

Fernández, M. A., López, M., López, E., Gutiérrez, D., Martínez, A., \& Pantoja, C. A. (2018). Educación en salud, práctica de actividad física y alimentación en grandes urbes: perspectiva de los usuarios. Revista Salud UIS, 50(2), 116-125.

García, C., Félix, R. O., Mercado, S. M., Sabag, E., García, R., Mejía, D. y Casanova, A. P. (2018). Asociación entre el síndrome de dolor miofascial y sintomatología ansiosa en docentes de educación superior. Revista Psicología y Salud, 28(1), 57-61.

García, C. M., \& Muñoz, A. I. (2013). Salud y trabajo de docentes de instituciones educativas distritales de la localidad uno de Bogotá. Avances en enfermería, 31(2), 30-42.

Gardner, S. (2010). Stress among prospective teachers: A review of the literature. Australian Journal of Teacher Education, 35(8), 2. 
Gil-Monte, P. (2009). Algunas razones para considerar los riesgos psicosociales en el trabajo y sus consecuencias en la salud pública. Revista Española de Salud Pública, (83) 169-173.

Gómez, V., Perilla, L. E., \& Hermosa, A. M. (2015). Moderación de la relación entre tensión laboral y malestar de profesores universitarios: Papel del conflicto y la facilitación entre el trabajo y la familia. Revista Colombiana de Psicología, 24(1), 185-201.

Heredia, S. A., Morales, M. F., Infante, R., Sánchez, D., Páez, C., \& Gabini, S. (2018). Psychosocial risk factors in university teachers. Revista Espacios, 39(49).

Hernández, A. I., Ortega, R. P., y Reidl, L. M. (2012). Validación del instrumento de estrés laboral para médicos mexicanos. En-claves del pensamiento, 6(11), 113-129.

Irigoyen, F. D., y Martínez, S. (2015). Estímulos económicos, productividad y salud en docentes de la Universidad Autónoma Benito Juárez de Oaxaca. Salud de los Trabajadores, 23 (2), 127-136.

Izquierdo, M. (1998). Políticas y experiencias de evaluación de académicos. Colección Pedagógica Universitaria, 30, 35-78.

Jensen, C. D., Cushing, C. C., Aylward, B. S., Craig, J. T., Sorell, D. M., \& Steele, R. G. (2011). Effectiveness of motivational interviewing interventions for adolescent substance use behavior change: a meta-analytic review. Journal of consulting and clinical psychology, 79 (4), 433.

Juárez, A., \& Camacho, A. (2011). Reflexiones teórico-conceptuales de lo psicosocial en el trabajo. Universidad Autónoma del Estado de Morelos. Primera Edición. México, 113-129.

López-Segrera, F. (2015). Educación superior comparada: tendencias mundiales y de América Latina y Caribe. Avaliação: Revista da Avaliação da Educação Superior, 21(1).

López, J. A., Tenahua, I., Xicali, N., Morales, F. A., Torres, A., \& Posadas, G. (2017). Prevalencia de factores de riesgo cardiovascular en académicos universitarios de una institución pública de Oaxaca. Revista Mexicana de Enfermería Cardiológica, 24 (Esp), 12-16.

Macías-Hernández, J. C., Alcantar-Carrillo, O. E., Castro-Alcantar, M. G., Kasten-Monges, M. J., y Cambero-González, E. G. (2018). Factores de riesgo para Hipertensión Arterial Sistémica y Diabetes Mellitus Tipo 2 en el personal docente de uno de los Departamentos del Centro Universitario de Ciencias de la Salud. Universidad de Guadalajara, febreromayo 2016. Salud Jalisco, 4(2), 81-90.

Martínez, C., Martínez, S., \& Méndez, I. (2015). Estímulos económicos, exigencias de trabajo y salud en académicos universitarios mexicanos. Salud de los Trabajadores, 23(1), 5-18.

Martínez-Hernández, A., Enríquez, L., Moreno-Moreno M. J., \& Martí, A. (2007). Genetics of obesity. Public Health Nutrition 10 (10A), 1138-1144.

Martínez, J. E., Tobón, S., \& Romero, A. (2017). Problemáticas relacionadas con la acreditación de la calidad de la educación superior en América Latina. Innovación educativa (México, DF), 17(73), 79-96.

Martínez, L., Vega, C., Nava, C., \& Anguiano, S. (2010). Revisión de investigaciones sobre las enfermedades del siglo XXI en México. Revista Electrónica de Psicología Iztacala, 13 (1) 186-202.

Martínez, S., \& Preciado, M. D. L. (2009). Consecuencias de las políticas neoliberales sobre el trabajo y la salud de académicos universitarios: el burnout como fenómeno emergente. Psicología y salud, 19 (2), 197-206.

Millán, A., Calvanese, N., \& D'Aubeterre, M. E. (2017). Condiciones de trabajo, estrés laboral, dependencia universitaria y bienestar psicológico en docentes universitarios. REDU. Revista de Docencia Universitaria, 15 (1), 195-218.

Muñoz, H. (2019). La burocracia universitaria. Revista de la Educación Superior, 48(189), 7396.

Olmedo, B., Delgado, I., López, M., Yáñez, J., Mora, A., Velasco, R., \& Montero, S. (2013). Perfil de salud en profesores universitarios y su productividad. Revista Cubana de Investigaciones Biomédicas, 32(2), 130-138.

Organización de las Naciones Unidas para la Educación. (1998). Declaración mundial sobre la educación superior en el siglo xxi: visión y acción. Recuperado de http://www.unesco.org/education/educprog/wche/declaration_spa.htm 
Organización de las Naciones Unidas para la Educación. (2009). Conferencia Mundial sobre la Educación Superior - 2009: La nueva dinámica de la educación superior y la investigación para el cambio social y el desarrollo. Recuperado de http://www.unesco.org/education/WCHE2009/comunicado_es.pdf

Palacios, M. E., \& Montes de Oca, V. (2017). Condiciones de Trabajo y Estrés en Académicos Universitarios. Ciencia \& trabajo, 19(58), 49-53.

Riley, R. D., Moons, K. G., Snell, K. I., Ensor, J., Hooft, L., Altman, D. G., Altman, D. G., Hayden, J. Collins, G. S., \& Debray, T. P. (2019). A guide to systematic review and metaanalysis of prognostic factor studies. BMJ, 364, k4597.

Rodríguez-Vega, M. C., Preciado, M. L., Aguilar-Aldrete, M. E., Aranda-Beltrán, C., LeónCortés, S., \& Franco, S. A. (2018). Causas y situaciones que inciden en el estrés laboral de profesores del CUCS-U DE G. Salud Jalisco, 1(2), 94-100.

Sánchez, C., \& Martínez, S. (2014). Condiciones de trabajo de docentes universitarios, satisfacción, exigencias laborales y daños a la salud. Salud de los Trabajadores, 22(1), 1928.

Sánchez, E. (2017). Retos de la educación superior en América Latina: el caso de República Dominicana. Ciencia y Sociedad, 42(1), 9-23.

Suárez, M. H., y Muñoz, H. (2016). ¿Qué pasa con los académicos? Revista de la educación superior, 45(180), 1-22.

Taracena, E. (2011). La productividad como criterio para evaluar el trabajo intelectual y docente sus consecuencias en la calidad del lazo social en la universidad. En: de Garay, G. (Coord.). Para estudiar el tiempo presente. Aproximaciones teórico-metodológicas y experiencias empíricas. México: Instituto Mora. En prensa.

Torres-Fonseca, A., \& López-Hernández, D. (2014). Criterios para publicar artículos de revisión sistemática. Revista de Especialidades Médico-Quirúrgicas, 19(3), 393-399.

Torres, C. A., \& Schugurensky, D. (2002). The political economy of higher education in the era of neoliberal globalization: Latin America in comparative perspective. Higher Education, 43(4), 429-455.

Tünnermann, C. (2008). La educación superior en América Latina y el Caribe: diez años después de la Conferencia Mundial de 1998. Pontificia Universidad Javeriana.

Tünnermann, C. (2010). Las conferencias regionales y mundiales sobre educación superior de la UNESCO y su impacto en la educación superior de América Latina. Universidades, 60 (47).

Von Elm, E., Altman, D. G., Egger, M., Pocock, S. J., Gøtzsche, P. C., y Vandenbroucke, J. P. (2008). Declaración de la Iniciativa STROBE (Strengthening the Reporting of Observational studies in Epidemiology): directrices para la comunicación de estudios observacionales. Gaceta Sanitaria, 22 (2), 144-150.

Watts, J., \& Robertson, N. (2011). Burnout in university teaching staff: a systematic literature review. Educational Research, 53 (1), 33-50.

Wilches-Luna, E. C., Hernández, N. L., Chavarro, P. A., y Bernal-Sánchez, J. J. (2016). Perfiles de riesgo cardiovascular y condición física en docentes y empleados no docentes de una facultad de salud. Revista de Salud Pública, 18, 890-903. 